and George Hull, then at Bell Laboratories, measured the thermal conductivity of various isotopic compositions of bulk germanium and found them to be significantly different ${ }^{3}$. More recently, the end of the Cold War resulted in a number of laboratories in the former Soviet Union making a variety of stable isotopes including isotopes of germanium, silicon, gallium and many other semiconductors available for peaceful research purposes. These isotopes have been used to explore various aspects of state-of-the-art silicon CMOS device fabrication (for example, ${ }^{30} \mathrm{Si}$ has been used to probe the time dependence of chemical reactions and impurity diffusion at interfaces). In parallel, much effort has been made to identify the effect of isotopic composition and randomness on a variety of electrical, magnetic, thermal and optical properties of semiconductors ${ }^{4,5}$. However, despite their scientific importance, isotope effects in silicon and germanium have had very little impact on the development of new semiconductor products. Isotope effects in diamond, on the other hand, may be different.

Diamond is well known for being 'number one' for properties such as hardness, thermal conductivity, bandwidth for optical transmission and chemical stability. Even though it is considered to be an insulator, recent advances in doping and lithography technology have made diamond an attractive material for the fabrication of high-power semiconductor devices. Thanks to its wide bandgap of $\sim 5.5 \mathrm{eV}$ (compared with $1.1 \mathrm{eV}$ for silicon and $1.43 \mathrm{eV}$ for gallium arsenide), its various optical emissions are observable at room temperature, and a number of groups around the world are exploring the possible use of diamond-based devices for applications in quantum information.

Isotope engineering of bulk diamond was independently pioneered by Hisao Kanda of the National Institute for Research in Inorganic Materials and colleagues in Japan ${ }^{6}$ and Tom Anthony of General Electric and co-workers in the US ${ }^{7}$. However, atomic layer-by-layer stacking of ${ }^{12} \mathrm{C}$ and ${ }^{13} \mathrm{C}$ isotopes has been extremely challenging because the microwave plasma-assisted chemical vapour deposition process that is needed to obtain high-quality diamond films involves simultaneous etching and growth processes at the substrate surface. Watanabe and co-workers successfully optimized the competition between etching and growth to perform atomic-layer-level control of the growth of ${ }^{12} \mathrm{C}$ and ${ }^{13} \mathrm{C}$ diamond films for the first time.

Watanabe and co-workers confirmed that electrons and holes were preferentially confined in the ${ }^{12} \mathrm{C}$ layers of the ${ }^{12} \mathrm{C} /{ }^{13} \mathrm{C}$ structures at temperatures of $77 \mathrm{~K}$ by detecting electroluminescence. The charge carriers were confined in the ${ }^{12} \mathrm{C}$ layers because the bandgap of ${ }^{12} \mathrm{C}$ is $17 \mathrm{meV}$ smaller than that of ${ }^{13} \mathrm{C}$. This is the first example of isotope engineering of a semiconductor's electronic and optical properties that might have a major impact on real-world applications, such as solidstate lasers composed exclusively of diamond that operate at extreme ultraviolet wavelengths (Fig. 1).

An important challenge for the future is to work with the $17 \mathrm{meV}$ difference between the bandgaps of ${ }^{12} \mathrm{C}$ and ${ }^{13} \mathrm{C}$ : although this is large for an isotope effect, it is still too small for room-temperature operations. However, by combining isotope engineering in diamond with another wide-bandgap semiconductor, such as silicon carbide, it may be possible to develop electronic and optical devices that can work at room temperature.

Kohei M. Itoh is at the Department of Applied Physics and Physico-Informatics, Keio University, Yokohama 223-8522, Japan.

e-mail:kitoh@appi.keio.ac.jp

\section{References}

1. Kroemer, H. Jpn. J. Appl. Phys. Suppl. 20-1, 9-13 (1981).

2. Watanabe, H., Nebel, C. E. \& Shikata, S. Science

324, 1425-1428 (2009)

3. Geballe, T. H. \& Hull, G. Phys. Rev. 110, 773-775 (1958).

4. Haller, E. E. J. Appl. Phys. 77, 2857-2878 (1995).

5. Cardona. M. \& Thewalt, M. L. W. Rev. Mod. Phys 77, 1173-1224 (2005).

6. Hanzawa, H. et al. Phys. Rev. B 54, 3793-3799 (1996).

7. Hass, K. C., Tamor, M. A., Anthony, T. R. \& Banholzer, W. F. Phys. Rev. B 44, 12046-12049 (1991).

\title{
CARBON NANOTUBES
}

\section{Sorted by DNA}

A single-walled carbon nanotube can be made by rolling up a hexagonal sheet of carbon atoms along any one of a number of different directions. Each of these directions is associated with a pair of numbers (known as the chirality) that determines the diameter and electronic properties of the nanotube. However, nanotubes are usually produced as a mixture of chiralities with one-third being metallic and twothirds being semiconducting. Although a number of methods for separating semiconducting and metallic nanotubes have been developed, it has proven more difficult to isolate nanotubes of a given chirality. Now Xiaomin Tu, Suresh Manohar, Anand Jagota and Ming Zheng of DuPont and Lehigh University have shown that DNA can be used to do just this (Nature 460, 250-253; 2009).

Zheng and co-workers exploited the fact that certain short strands of DNA will bind together in sheets and then fold around nanotubes of a particular chirality to form a barrel shape. Once the DNA had bound to the nanotubes, the different types could be separated by ion-exchange chromatography. This process yielded single-chirality samples with up to $90 \%$ purity. The figure shows the optical absorption spectra for a mixture of nanotubes ( $\mathrm{HiPco}$ ) and 12 different semiconducting nanotubes: the structures of the nanotubes (viewed along their axis) are shown on the right. The spectra are ordered according to the position of the $E_{11}$ absorption peak.

This purification process could, in principle, be scaled up. However, this process is expensive, so instead it may be used to provide purified 'seed' nanotubes that could act as templates for growing more nanotubes with the same chirality.

TIM REID

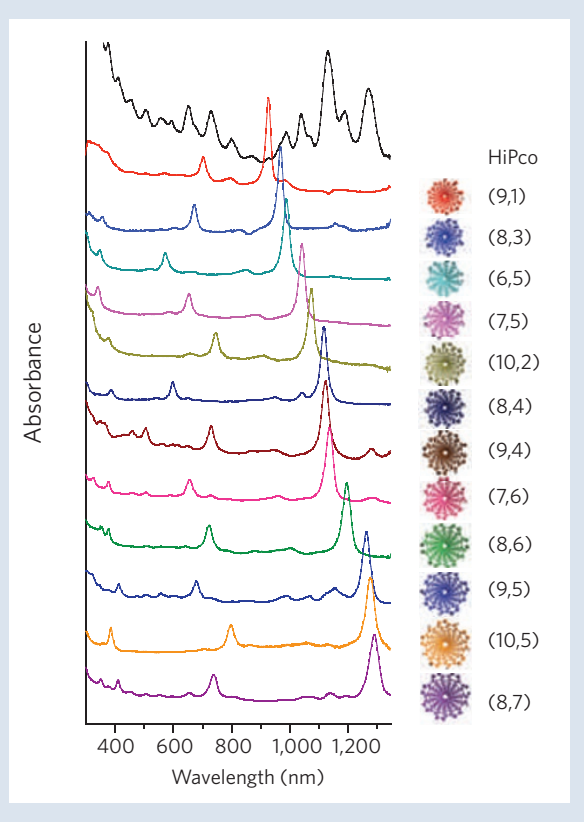

